\title{
Associations between Maternal and Fetal Inherited Thrombophilias, Placental Characteristics Associated with Vascular Malperfusion, and Fetal Growth
}

\author{
Alexa A. Freedman ${ }^{1}$ Carol J. Hogue ${ }^{1}$ Donald J. Dudley ${ }^{2}$ Robert M. Silver ${ }^{3}$ Barbara J. Stoll ${ }^{4}$ \\ Halit Pinar ${ }^{5}$ Robert L. Goldenberg ${ }^{6}$ Carolyn Drews-Botsch ${ }^{1}$
}

${ }^{1}$ Department of Epidemiology, Rollins School of Public Health, Emory University, Atlanta, Georgia, United States

2 Department of Obstetrics and Gynecology, School of Medicine, University of Virginia, Charlottesville, Virginia, United States

${ }^{3}$ Department of Obstetrics and Gynecology, School of Medicine, University of Utah, Salt Lake City, Utah, United States

${ }^{4}$ McGovern Medical School, University of Texas Health Science Center, Houston, Texas, United States

${ }^{5}$ Department of Pathology and Laboratory Medicine, Alpert Medical

School, Brown University, Providence, Rhode Island, United States

6 Department of Obstetrics and Gynecology, Columbia University

Medical Center, New York, New York, United States

\author{
Address for correspondence Alexa A. Freedman, Department of \\ Epidemiology, Rollins School of Public Health, Emory University, \\ 1518 Clifton Road, Atlanta, GA 30322, United States \\ (e-mail: alexa.freedman@emory.edu).
}

TH Open 2017;1:e43-e55.

\begin{abstract}
Keywords

- pregnancy

- inherited thrombophilias

- perinatal hemostasis

Pregnancy results in alterations in coagulation processes, which may increase the risk of thrombosis. Inherited thrombophilia mutations may further increase this risk, possibly through alterations in the placenta, which may result in pregnancy complications such as poor fetal growth. The purpose of our study is to evaluate the association of fetal growth, approximated by birth weight for gestational age percentile, with genetic markers of thrombophilia and placental characteristics related to vascular malperfusion. We analyzed data from the Stillbirth Collaborative Research Network's population-based case-control study conducted in 2006-2008. Study recruitment occurred in five states: Rhode Island and counties in Massachusetts, Georgia, Texas, and Utah. The analysis was restricted to singleton, nonanomalous live births $\leq 42$ weeks' gestation with a complete placental examination and successful testing for $\geq 1$ thrombophilia marker (858 mothers, 902 infants). Data were weighted to account for oversampling, differential consent, and availability of placental examination. We evaluated five thrombophilia markers: factor $\mathrm{V}$ Leiden, factor II prothrombin, methylenetetrahydrofolate reductase $\mathrm{A} 1298 \mathrm{C}$ and $\mathrm{C} 677 \mathrm{~T}$, and plasminogen activator inhibitor type 1 in both maternal blood and placenta/cord blood. We modeled maternal and fetal thrombophilia markers separately using linear regression. Maternal factor $\mathrm{V}$ Leiden mutation was associated with a 13.16-point decrease in adjusted birth weight percentile (95\% confidence interval: $-25.50,-0.82$ ). Adjustment for placental abnormalities related to vascular malperfusion did not affect the observed association. No other maternal or fetal thrombophilia markers were significantly associated with birth weight percentile. Maternal factor $V$ Leiden may be associated with fetal growth independent of placental characteristics.
\end{abstract}

(c) 2017 Georg Thieme Verlag KG Stuttgart · New York 


\section{Introduction}

Physiologic changes during pregnancy include alterations in coagulation processes in the preparation for potential bleeding at the time of delivery. ${ }^{1}$ These physiologic changes also increase the risk of thrombosis. ${ }^{1}$ Presence of an inherited thrombophilic mutation may further increase this risk and may be associated with pregnancy complications. Inherited thrombophilias include mutations that affect the coagulation cascade, which can increase the risk of venous thromboembolism (VTE). ${ }^{2}$ Some of the more common mutations resulting in inherited thrombophilia include factor $\mathrm{V}$ Leiden (FV Leiden), factor II prothrombin (FII prothrombin), methylenetetrahydrofolate reductase (MTHFR), and plasminogen activator inhibitor type 1 (PAI-1). ${ }^{2}$ Currently, the American College of Obstetricians and Gynecologists recommends screening for inherited thrombophilias only when a pregnant woman has a history of VTE or a first-degree relative with thrombophilia due to insufficient evidence linking inherited thrombophilias with pregnancy complications. ${ }^{3}$

Several studies have evaluated the relationship between inherited thrombophilias and fetal growth restriction, although results have been inconclusive. A systematic review published in 2005 reported that both FV Leiden and FII prothrombin are associated with an increased risk of fetal growth restriction; however, the authors caution that this association may be driven by low-quality studies with extreme associations. ${ }^{4}$ A more recent systematic review of FV Leiden reported an association between maternal FV Leiden and small-for-gestational-age (SGA) infant. ${ }^{5}$ Recent individual studies have reported conflicting results. A casecontrol study in Italy reported no association between FV Leiden, FII prothrombin, or MTHFR C677T and fetal growth restriction, determined from abnormal umbilical arterial blood flow. ${ }^{6}$ Conversely, a similar, larger study also conducted in Italy reported that FV Leiden and FII prothrombin mutations in neonates, but not mothers, are associated with increased risk of fetal growth restriction in those without hypertension. $^{7}$ MTHFR mutations are associated with hyperhomocysteinemia, ${ }^{8}$ which has been associated with fetal growth. ${ }^{9}$ Conflicting results have been reported for PAI- $1 .^{10-12}$ However, a recent case report suggests that this mutation is an independent risk factor for placental insufficiency. $^{13}$

Potential associations between inherited thrombophilia and fetal growth restriction may be mediated by alterations in placental function, as the placenta is responsible for regulating fetal growth and development through mediating oxygen, nutrient, and waste transfer. The purpose of our study is to evaluate the association of fetal growth, approximated by birth weight for gestational age percentile, with genetic markers of thrombophilia and placental characteristics related to vascular malperfusion. We hypothesize that the relationship between placental characteristics related to vascular malperfusion and fetal growth may be driven in part by maternal and/or fetal thrombophilic mutations.

\section{Materials and Methods}

The Stillbirth Collaborative Research Network (SCRN) conducted a population-based case-control study of stillbirth. Participants delivering a stillborn or live born infant were enrolled between March 2006 and September 2008. Recruitment occurred at 59 hospitals representing five catchment areas of the United States: Rhode Island and counties in Massachusetts, Georgia, Texas, and Utah. Controls were sampled from all live births in these catchment areas using a stratified random method. The SCRN study enrolled 663 women with a stillbirth (cases) and 1,932 women with a live birth (controls). Details of the study design, including sampling methods, have been published elsewhere. ${ }^{14}$ The study was approved by the Institutional Review Board at each study site.

Birth weight and gestational age were obtained from medical record abstraction. Birth weight percentile adjusted for gestational age was determined from Hadlock's growth curves. ${ }^{15}$ We conducted a secondary analysis using logistic regression with SGA, defined as a birth weight less than 10th percentile for gestational age, as the outcome. Women had the option to consent to a placental examination, which was conducted by a perinatal pathologist using a standard protocol. ${ }^{16}$ of the 1,932 women with a live birth, $93.4 \%$ consented to a placental examination that was completed and considered adequate in $69.7 \%{ }^{16}$ In $23.1 \%$ of those who consented to a placental examination, the placenta had been previously discarded and could not be examined. ${ }^{16}$ The presence of placental characteristics related to maternal and fetal vascular malperfusion was determined from this examination. Characteristics potentially related to maternal vascular malperfusion include retroplacental hematoma, any parenchymal infarction (focal, multifocal, diffuse), intraparenchymal thrombus, and perivillous/intervillous fibrin/fibrinoid deposition. Characteristics related to fetal vascular malperfusion include fetal vascular thrombi in the chorionic plate, any avascular villi (focal, multifocal, diffuse), and edema (placental hydrops).

Samples were collected at the time of birth and stored at $-80^{\circ} \mathrm{C}$ prior to assay. Testing for thrombophilia markers occurred 2 to 5 years after sample collection. Maternal thrombophilia markers were evaluated using maternal blood and fetal thrombophilia markers were evaluated using fetal blood from the umbilical cord and placental tissue. Samples were genotyped for four single nucleotide polymorphisms (FV Leiden, rs6025; prothrombin G20210A, rs1799963; MTHFR C677T, rs1801133; and MTHFR A1298C, rs1801131) and one deletion/insertion polymorphism (PAI 4G/5G, rs1799768) using TaqMan allelic discrimination chemistry (Life Technologies, Foster City, California, United States) with validated assays and the manufacturer's protocols. Specifically, 6 ng of DNA was included in a $5-\mu \mathrm{L}$ reaction with a final concentration of $\times 1$ TaqMan genotyping master mix and $\times 0.5$ TaqMan genotyping assay mix. Details of DNA extraction and evaluation have been reported. ${ }^{12}$ Genotypes were verified and the polymorphisms were evaluated for deviation from Hardy-Weinberg equilibrium. All samples were genotyped in duplicate to ensure 
accurate genotyping. Laboratory personnel were blinded to the clinical status of the samples. ${ }^{12}$

We restricted our analysis to live births (controls) with a placental examination and successful thrombophilia testing for at least one thrombophilia maker in either maternal blood (mother) or placenta/cord blood (infant). We additionally excluded multiple gestations, anomalous pregnancies, and births more than 42 weeks' gestation. Our final sample included 858 mothers and 902 infants with successful testing for at least one marker (-Fig. 1).

Data weights were calculated to account for differential consent for enrolment, oversampling, and availability of the placental examination. ${ }^{14}$ Weights for the availability of the placental examination incorporated clinic site, induction, time trend, delivery time, weekend delivery, and mode of delivery. We conducted weighted linear regression to evaluate the association of fetal growth, approximated by birth weight for gestational age percentile, with thrombophilia markers and placental characteristics. Analyses were conducted separately for maternal (the presence of thrombophilia markers in maternal blood with placental characteristics related to maternal vascular malperfusion) and infant (the presence of thrombophilia markers in placenta/cord blood with placental characteristics related to fetal vascular malperfusion) throm-

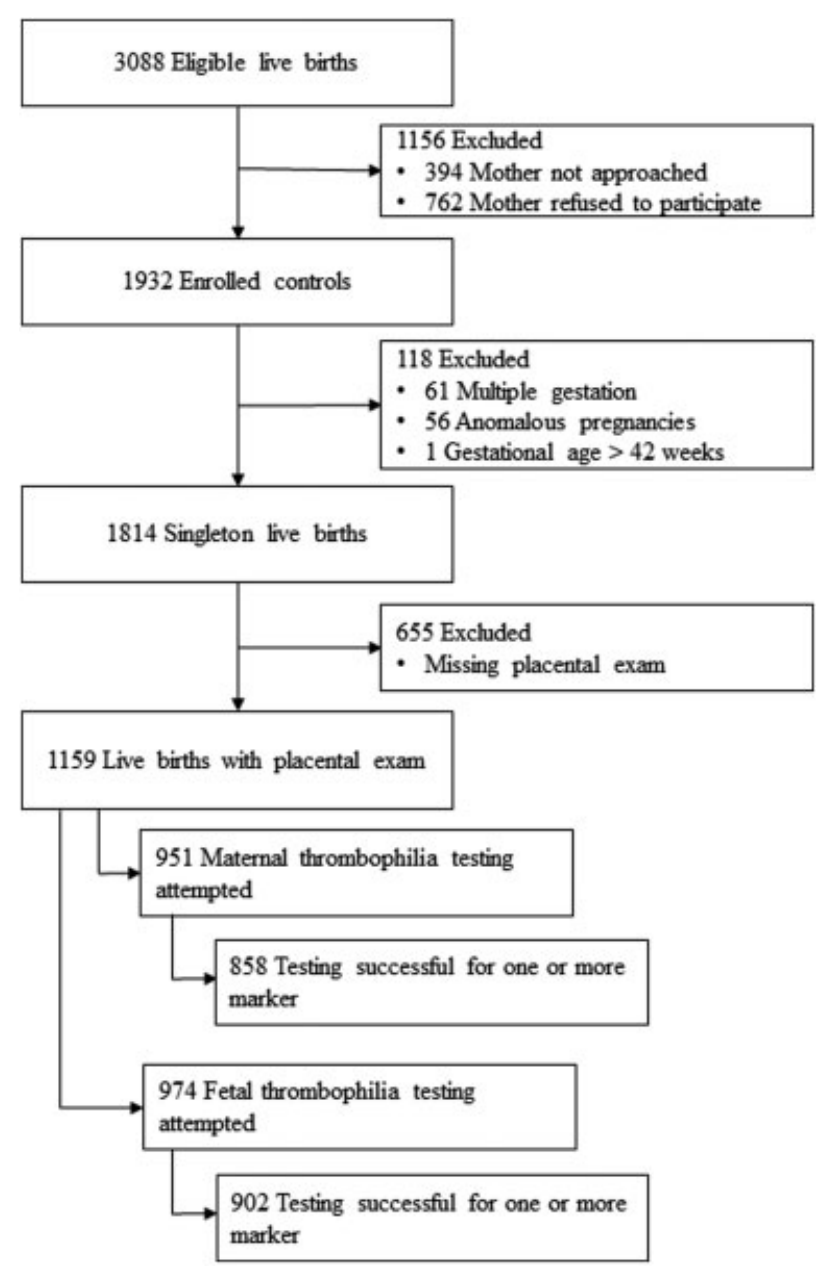

Fig. 1 Study enrollment and inclusion. bophilia. Each of the five thrombophilia markers of interest was classified as present (individuals with one or two copies of the mutation) or absent (individuals with no copies of the mutation-common homozygous). We also created a summary variable to indicate number of thrombophilia markers present and analyzed this as a dichotomous variable (no markers present vs. one or more markers present). We controlled for maternal age and maternal race/ethnicity (selfreported) in the maternal and fetal models and additionally controlled for paternal age and paternal race/ethnicity in the fetal models, as these characteristics may confound the association between presence of thrombophilia markers and fetal growth. To evaluate differences in means and proportions, $t$-tests and chi-square tests were used, respectively. All statistical tests used a $p$-value of less than 0.05 to determine statistical significance. Analyses were performed using SUDAAN version 11.0 (Research Triangle Institute, Research Triangle Park, North Carolina, United States). ${ }^{17}$

\section{Results}

\section{Descriptive Statistics}

Our analytic population consisted of predominantly term births (91.6\%; - Table 1). The average birth weight for gestational age percentile was 42.99 , with $71.0 \%$ of infants falling between the 10th and 90th percentiles. Roughly, three-quarters of mothers were between 20 and 34 years of age and $46.0 \%$ were non-Hispanic white. Similarly, $70.0 \%$ of fathers were between 20 and 34 years of age and $43.1 \%$ were non-Hispanic white. Over half of mothers had completed at least 1 year of college and $63.1 \%$ were married at the time of birth. Approximately half of mothers had a pre-pregnancy body mass index within the normal range (18.5-24.9) and $87.2 \%$ of mothers did not smoke in the 3 months prior to pregnancy.

\section{Thrombophilia Markers and Fetal Growth}

As expected, FV Leiden and FII prothrombin were relatively rare in this population ( 2.8 and $1.9 \%$ in mothers and 2.6 and $1.5 \%$ in infants, respectively; - Table 2 ). The other markers were more common (PAI-1: 62.2\% in mothers, $61.3 \%$ in infants; MTHFR A1298C: $38.5 \%$ in mothers and 40.3\% in infants; MTHFR C677T: $55.5 \%$ in mothers and $54.2 \%$ in infants); $82.6 \%$ of mothers and $84.6 \%$ of infants had at least one marker. Maternal FV Leiden was associated with a lower mean birth weight percentile in crude analyses (absent: 43.20, present: 33.74), although this difference was not statistically significant. Conversely, those with fetal FV Leiden had a higher mean birth weight percentile (absent: 42.72, present: 49.20). Other thrombophilic polymorphisms were not associated with lower mean birth weight percentile, and the maternal PAI-1 genotype was associated with a statistically significantly higher mean birth weight percentile (absent: 38.85, present: 44.36).

\section{Placental Characteristics and Fetal Growth}

With the exception of parenchymal infarction, which occurred in $16.2 \%$ of pregnancies, and intraparenchymal thrombus, which occurred in $13.6 \%$ of pregnancies, placental changes related to thrombosis were rare (-Table 3 ). For 
Table 1 Descriptive characteristics of study population

\begin{tabular}{|c|c|}
\hline \multirow[t]{3}{*}{ Characteristics } & Mean (SE) or $\%_{w}$ \\
\hline & $N=1,073$ \\
\hline & $N_{\mathrm{w}}=882$ \\
\hline Adjusted birth weight percentile ${ }^{a}$ & $42.99(1.01)$ \\
\hline$<5$ th percentile & 8.6 \\
\hline 5th-10th percentile & 6.4 \\
\hline 10th-90th percentile & 71.0 \\
\hline 90th-95th percentile & 5.7 \\
\hline 95th-100th percentile & 8.3 \\
\hline \multicolumn{2}{|l|}{ Gestational age } \\
\hline$<20$ completed wk & 0.0 \\
\hline 20-23 completed wk & 0.3 \\
\hline 24-27 completed wk & 0.4 \\
\hline 28-31 completed wk & 0.6 \\
\hline 32-36 completed wk & 7.2 \\
\hline$\geq 37$ completed wk & 91.6 \\
\hline \multicolumn{2}{|l|}{ Maternal age } \\
\hline$<20$ & 9.9 \\
\hline $20-34$ & 76.2 \\
\hline $35-39$ & 11.4 \\
\hline $40+$ & 2.5 \\
\hline \multicolumn{2}{|l|}{ Paternal age } \\
\hline$<20$ & 5.3 \\
\hline $20-34$ & 70.0 \\
\hline $35-39$ & 17.3 \\
\hline $40+$ & 7.3 \\
\hline \multicolumn{2}{|l|}{ Maternal race/ethnicity } \\
\hline Non-Hispanic white & 46.0 \\
\hline Non-Hispanic black & 10.2 \\
\hline Hispanic & 36.9 \\
\hline Other & 6.9 \\
\hline \multicolumn{2}{|l|}{ Paternal race/ethnicity } \\
\hline Non-Hispanic white & 43.1 \\
\hline Non-Hispanic black & 10.9 \\
\hline Hispanic & 38.0 \\
\hline Other & 8.0 \\
\hline \multicolumn{2}{|l|}{ Maternal education } \\
\hline $\begin{array}{l}0-11 \text { (none/primary/some } \\
\text { secondary) }\end{array}$ & 17.7 \\
\hline 12 (completed secondary) & 27.1 \\
\hline $13+($ college $)$ & 55.2 \\
\hline \multicolumn{2}{|l|}{ Marital status } \\
\hline Not married or cohabitating & 14.8 \\
\hline Cohabitating & 22.2 \\
\hline Married & 63.1 \\
\hline
\end{tabular}

Table 1 (Continued)

\begin{tabular}{|c|c|}
\hline \multirow[t]{3}{*}{ Characteristics } & Mean (SE) or $\%_{w}$ \\
\hline & $N=1,073$ \\
\hline & $N_{\mathrm{w}}=882$ \\
\hline \multicolumn{2}{|l|}{ Maternal BMI } \\
\hline$<18.5$ & 3.0 \\
\hline $18.5-24.9$ & 51.2 \\
\hline $25-29.9$ & 22.7 \\
\hline $30-34.9$ & 12.5 \\
\hline$\geq 35$ & 10.6 \\
\hline \multicolumn{2}{|l|}{ Insurance } \\
\hline No insurance & 4.2 \\
\hline Any public/private insurance & 47.8 \\
\hline $\begin{array}{l}\text { VA/commercial health } \\
\text { insurance/HMO }\end{array}$ & 48.1 \\
\hline \multicolumn{2}{|l|}{ Maternal smoking status ${ }^{\mathrm{b}}$} \\
\hline Did not smoke & 87.2 \\
\hline$<10$ & 6.3 \\
\hline$\geq 10$ & 6.5 \\
\hline \multicolumn{2}{|l|}{ Alcohol use ${ }^{c}$} \\
\hline Did not drink & 58.8 \\
\hline Drank, no binging & 22.9 \\
\hline Binged & 18.3 \\
\hline \multicolumn{2}{|l|}{ Illicit drug use ${ }^{d}$} \\
\hline Never used drugs & 72.2 \\
\hline Ever used drugs, no addiction & 25.7 \\
\hline Ever used drugs, addiction & 2.1 \\
\hline
\end{tabular}

Abbreviations: \%, percent of weighted population; BMI, body mass index; $\mathrm{HMO}$, health maintenance organizations; $\mathrm{N}_{\mathrm{w}}$, weighted sample size; SE, standard error; VA, Veterans Affairs.

Note: Study population includes singleton, nonanomalous live births $\leq 42$ weeks' gestation with successful testing for at least one maternal or fetal thrombophilia marker.

aBirth weight for gestational age percentiles determined from Hadlock's norms.

${ }^{\mathrm{b}}$ Average number of cigarettes during 3 months prior to pregnancy.

'Alcohol consumption during 3 months prior to pregnancy.

dLifetime drug use.

example, retroplacental hematoma was observed in $4.4 \%$ of pregnancies, and perivillous/intervillous fibrinoid deposition occurred in $1.5 \%$ of pregnancies. Intraparenchymal thrombus was significantly associated with a higher mean birth weight percentile (present: 51.47, absent: 41.55). Conversely, perivillous/intervillous fibrin/fibrinoid deposition was significantly associated with a lower mean birth weight percentile (present: 26.67, absent: 42.84). The prevalence of markers of fetal vascular malperfusion (fetal vascular thrombi in the chorionic plate and avascular villi) were observed in approximately $7 \%$ of examined placentas. Edema was present in less than $1 \%$ of examined placentas. None of 
Table 2 Birth weight for gestational age percentile by presence of thrombophilia marker

\begin{tabular}{|c|c|c|c|}
\hline Thrombophilia marker (common homozygous vs. other) & $\mathrm{N}_{\mathrm{w}}$ or $\%_{\mathrm{w}}$ & $\begin{array}{l}\text { Percentile } \\
\text { Mean (SE) }\end{array}$ & $p$-Value ${ }^{a}$ \\
\hline \multicolumn{4}{|l|}{ FV Leiden G508A (rs6025) } \\
\hline Maternal marker & 638 & & \\
\hline GG & 97.2 & $43.20(1.22)$ & \multirow[t]{2}{*}{0.14} \\
\hline Other & 2.8 & $33.74(6.32)$ & \\
\hline Fetal marker & 668 & & \\
\hline GG & 97.4 & $42.72(1.14)$ & \multirow[t]{2}{*}{0.35} \\
\hline Other & 2.6 & $49.20(6.78)$ & \\
\hline \multicolumn{4}{|l|}{ FIl prothrombin G20210 (rs1799963) } \\
\hline Maternal marker & 644 & & \\
\hline GG & 98.1 & $42.63(1.21)$ & \multirow[t]{2}{*}{0.81} \\
\hline Other & 1.9 & $44.83(8.86)$ & \\
\hline Fetal marker & 668 & & \\
\hline GG & 98.5 & $43.03(1.13)$ & \multirow[t]{2}{*}{0.11} \\
\hline Other & 1.5 & $56.40(8.40)$ & \\
\hline \multicolumn{4}{|l|}{ PAl-1 4G/5G in/del (rs1799768) } \\
\hline Maternal marker & 592 & & \\
\hline $5 G / 5 G$ & 37.8 & $38.85(1.81)$ & \multirow[t]{2}{*}{0.03} \\
\hline Other & 62.2 & $44.36(1.65)$ & \\
\hline Fetal marker & 661 & & \\
\hline $5 G / 5 G$ & 38.7 & $41.78(1.75)$ & \multirow[t]{2}{*}{0.41} \\
\hline Other & 61.3 & $43.66(1.47)$ & \\
\hline \multicolumn{4}{|l|}{ MTHFR A1298C (rs1801131) } \\
\hline Maternal marker & 610 & & \\
\hline AA & 61.5 & $42.20(1.55)$ & \multirow[t]{2}{*}{0.79} \\
\hline Other & 38.5 & $42.85(1.94)$ & \\
\hline Fetal marker & 711 & & \\
\hline AA & 59.7 & $42.70(1.42)$ & \multirow[t]{2}{*}{0.74} \\
\hline Other & 40.3 & $43.42(1.69)$ & \\
\hline \multicolumn{4}{|l|}{ MTHFR C677T (rs1801133) } \\
\hline Maternal marker & 597 & & \\
\hline CC & 44.5 & $43.03(1.83)$ & \multirow[t]{2}{*}{0.74} \\
\hline Other & 55.5 & $42.19(1.67)$ & \\
\hline Fetal marker & 696 & & \\
\hline CC & 45.8 & $42.87(1.60)$ & \multirow[t]{2}{*}{0.91} \\
\hline Other & 54.2 & $43.13(1.52)$ & \\
\hline \multicolumn{4}{|l|}{ Summary marker variable } \\
\hline Maternal marker & 685 & & \\
\hline 0 markers & 17.4 & $43.55(2.74)$ & \multirow[t]{2}{*}{0.79} \\
\hline$\geq 1$ markers & 82.6 & $42.76(1.27)$ & \\
\hline Fetal marker & 749 & & \\
\hline 0 markers & 15.4 & $40.96(2.55)$ & \multirow[t]{2}{*}{0.41} \\
\hline$\geq 1$ markers & 84.6 & $43.28(1.16)$ & \\
\hline
\end{tabular}

Abbreviations: $\mathrm{N}_{\mathrm{w}}$, weighted sample size; \% $\%_{\mathrm{w}}$, percent of weighted population; SE, standard error.

Note: Thrombophilia markers (common homozygous vs. other) evaluated in maternal blood and placenta/cord blood in singleton, nonanomalous live births $\leq 42$ weeks' gestation, excluding those without a placental examination.

${ }^{a} p$-Values calculated using $t$-test for difference in means. 
Table 3 Birth weight for gestational age percentile by presence placental characteristics related to vascular malperfusion

\begin{tabular}{|c|c|c|c|}
\hline Placental characteristic & $\mathrm{N}_{\mathrm{w}}$ or $\%_{\mathrm{w}}$ & $\begin{array}{l}\text { Percentile } \\
\text { Mean (SE) }\end{array}$ & $p$-Value ${ }^{a}$ \\
\hline \multicolumn{4}{|l|}{ Maternal vascular malperfusion } \\
\hline Retroplacental hematoma & 878 & & \\
\hline Present & 4.4 & $47.61(4.23)$ & \multirow[t]{2}{*}{0.27} \\
\hline Absent & 95.6 & $42.82(1.04)$ & \\
\hline Parenchymal infarction & 879 & & \\
\hline Present & 16.2 & $39.66(2.17)$ & \multirow[t]{2}{*}{0.12} \\
\hline Absent & 83.8 & $43.51(1.13)$ & \\
\hline Intraparenchymal thrombus & 875 & & \\
\hline Present & 13.6 & $51.47(2.61)$ & \multirow[t]{2}{*}{0.00} \\
\hline Absent & 86.4 & $41.55(1.08)$ & \\
\hline Perivillous/intervillous fibrin/fibrinoid deposition & 846 & & \\
\hline Present & 1.5 & $26.67(5.88)$ & \multirow[t]{2}{*}{0.01} \\
\hline Absent & 98.5 & $42.84(1.04)$ & \\
\hline \multicolumn{4}{|l|}{ Fetal vascular malperfusion } \\
\hline Fetal vascular thrombi in the chorionic plate & 878 & & \\
\hline Present & 7.5 & $41.36(3.77)$ & \multirow[t]{2}{*}{0.68} \\
\hline Absent & 92.5 & $42.98(1.05)$ & \\
\hline Avascular villi & 878 & & \\
\hline Present & 7.3 & $42.02(3.55)$ & \multirow[t]{2}{*}{0.80} \\
\hline Absent & 92.7 & $42.94(1.05)$ & \\
\hline Edema (placental hydrops) & 879 & & \\
\hline Present & 0.9 & $31.22(8.62)$ & \multirow[t]{2}{*}{0.17} \\
\hline Absent & 99.1 & $43.02(1.02)$ & \\
\hline
\end{tabular}

Abbreviations: $\mathrm{N}_{\mathrm{w}}$, weighted sample size; $\%_{\mathrm{w}}$, percent of weighted population; SE, standard error.

Note: Restricted to singleton, nonanomalous live births $\leq 42$ weeks' gestation, excluding those with no successful thrombophilia testing.

${ }^{a} p$-Values calculated using $t$-test for difference in means.

the placental characteristics related to fetal vascular malperfusion were associated with a statistically significant difference in mean birth weight percentile.

Thrombophilia Markers and Placental Characteristics Although not statistically significant, the prevalence of an intraparenchymal thrombus was at least twice as common in placentas of women who had the FV Leiden mutation (present: 23.6\%, absent: 13.2\%) and in the placentas of infants who also had the FV Leiden mutation (present: $30.4 \%$, absent: 13.3\%; - Table 4). Similarly, avascular villi were more than twice as common if the mother or infant had the FV Leiden mutation. In contrast, perivillous/intervillous fibrin was significantly less common in pregnancies in which the mother or the infant had the FV Leiden mutation (maternal present: $0.2 \%$, absent: $1.8 \%$; fetal present: $0.0 \%$, absent: $1.5 \%$ ). Furthermore, fetal vascular thrombi in the chorionic plate and edema were significantly less frequent if the mother carried the FV Leiden mutation. FII prothrombin in mothers was associated with lower prevalence of parenchymal infarction, perivillous/intervillous fibrin, and fetal vascular thrombi in the chorionic plate, but a nonsignificant increase in the prevalence of edema. Other thrombophilia polymorphisms were generally not associated with differences in the prevalence of placental characteristics with the exception that fetal vascular thrombi were less common in mothers with PAI-1, perivillous/intervillous fibrin/fibrinoid deposition was more common in mothers with the MTHFR A1298C mutation, and parenchymal infarction was more common in mothers with the MTHFR C677T mutation.

\section{Analysis Results: Maternal Models}

When inherited thrombophilia was categorized as zero mutations present versus one or more mutations present (Models 1 and 3), there was no association between thrombophilia and adjusted birth weight percentile ( - Table 5 ). This was consistent regardless of whether or not the placental characteristics of interest were included in the model. In the models that included the placental characteristics (Models 1 and 2), parenchymal infarction and perivillous/intervillous fibrin/fibrinoid deposition were associated with a decrease in adjusted birth weight percentile, while intraparenchymal thrombus 


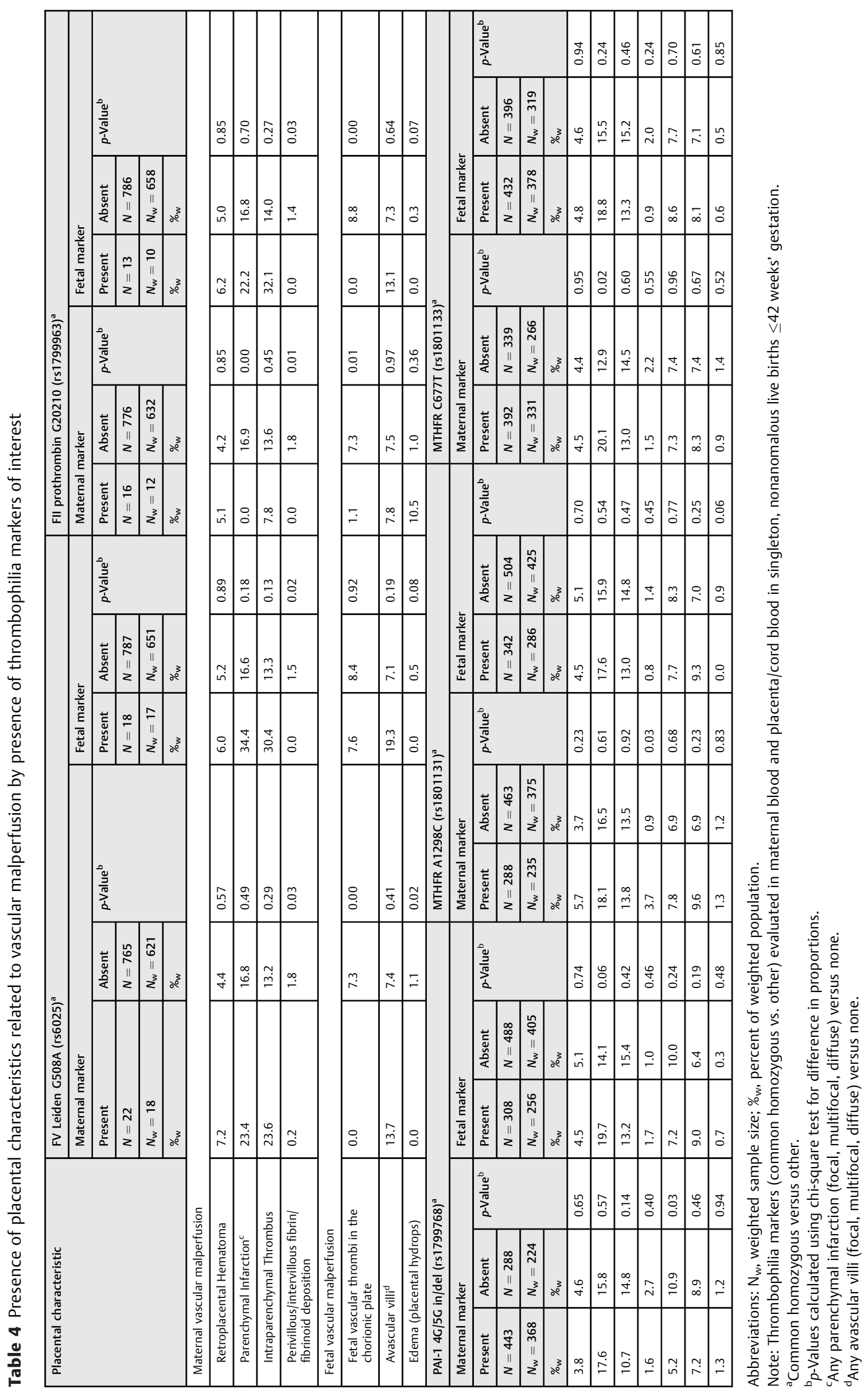



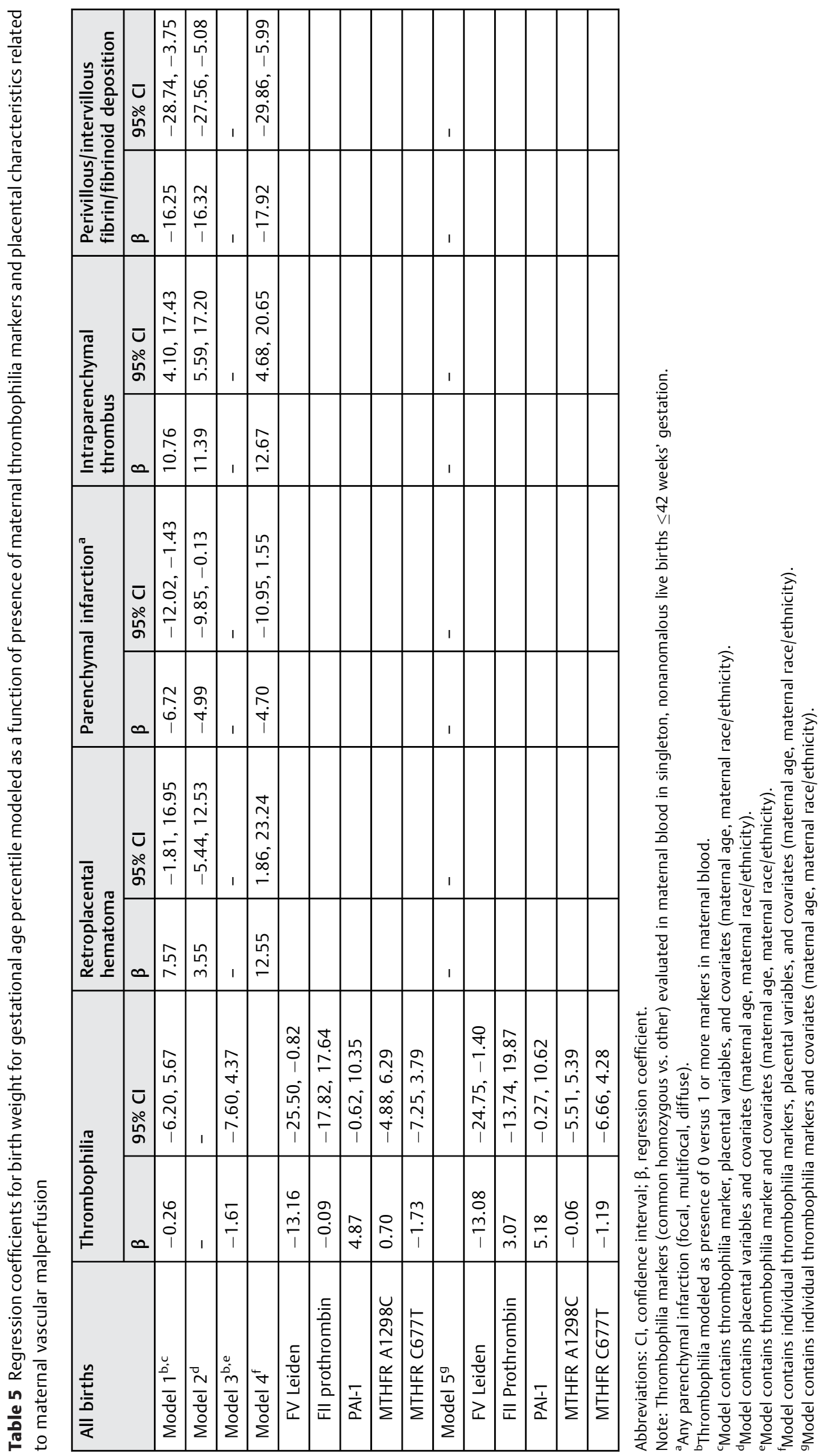
was associated with an increase in adjusted birth weight percentile. There was no association between retroplacental hematoma and adjusted birth weight percentile. These results were consistent regardless of whether or not thrombophilia was included in the model.

When each maternal thrombophilia mutation was included separately in the model (Models 4 and 5), FV Leiden was associated with a 13-point decrease in adjusted birth weight percentile regardless of whether or not placental characteristics were included in the model. There was no statistically significant association between any other inherited thrombophilia and adjusted birth weight percentile.

\section{Analysis Results: Fetal Models}

There was no statistically significant association between the summary thrombophilia variable or the individual thrombophilia mutations and adjusted birth weight percentile (-Table 6). These results were consistent in the models with and without adjustment for placental characteristics related to fetal vascular malperfusion. Similarly, none of the placental characteristics included in these models were significantly associated with adjusted birth weight percentile, regardless of adjustment for thrombophilia variables.

\section{Analysis Results: Small for Gestational Age}

When logistic regression was used to model the dichotomous SGA outcome, there were no statistically significant associations between the summary thrombophilia variable and the individual thrombophilia variables with SGA in the maternal or fetal models, regardless of whether or not placental characteristics were included in the model ( - Tables 7 and 8 ). In the fully adjusted maternal model, the odds ratio (OR) for the association between SGA and the FV Leiden mutation was 1.43 (95\% confidence interval [CI]: 0.33, 6.11; - Table 7). In the maternal models, intraparenchymal thrombus was consistently significantly associated with a protective effect against SGA (OR, fully adjusted model: 0.35; 95\% CI: 0.14, 0.87).

\section{Discussion}

Based on our results, we suggest that maternal FV Leiden may be associated with reduced birth weight percentile and

Table 6 Regression coefficients for birth weight for gestational age percentile modeled as a function of presence of fetal thrombophilia markers and placental characteristics related to fetal vascular malperfusion

\begin{tabular}{|c|c|c|c|c|c|c|c|c|}
\hline \multirow[t]{2}{*}{ All births } & \multicolumn{2}{|c|}{ Thrombophilia } & \multicolumn{2}{|c|}{$\begin{array}{l}\text { Fetal vascular thrombi } \\
\text { in the chorionic plate }\end{array}$} & \multicolumn{2}{|c|}{ Avascular villia } & \multicolumn{2}{|l|}{ Edema } \\
\hline & $\beta$ & $95 \% \mathrm{Cl}$ & $\beta$ & $95 \% \mathrm{Cl}$ & $\beta$ & $95 \% \mathrm{Cl}$ & $\beta$ & $95 \% \mathrm{Cl}$ \\
\hline Model $1^{\text {b,c }}$ & 0.56 & $-5.21,6.32$ & -5.47 & $-13.42,2.47$ & -0.55 & $-8.53,7.43$ & -3.63 & $-32.06,24.79$ \\
\hline Model $2^{\text {d }}$ & - & - & -2.83 & $-10.79,5.14$ & -1.52 & $-9.06,6.02$ & -6.89 & $-25.24,11.46$ \\
\hline Model $3^{\text {b,e }}$ & 0.58 & $-5.15,6.31$ & - & - & - & - & - & - \\
\hline Model $4^{f}$ & & & -5.09 & $-13.34,3.16$ & 0.03 & $-9.25,9.31$ & -5.05 & $-32.94,22.85$ \\
\hline FV Leiden & 5.55 & $-7.69,18.79$ & & & & & & \\
\hline FII Prothrombin & 5.85 & $-13.39,25.08$ & & & & & & \\
\hline PAI-1 & 1.36 & $-3.73,6.46$ & & & & & & \\
\hline MTHFR A1298C & -0.72 & $-5.98,4.53$ & & & & & & \\
\hline MTHFR C677T & -1.72 & $-7.14,3.69$ & & & & & & \\
\hline Model $5^{9}$ & & & - & - & - & - & - & - \\
\hline FV Leiden & 5.39 & $-8.18,18.95$ & & & & & & \\
\hline FII Prothrombin & 6.19 & $-12.83,25.20$ & & & & & & \\
\hline PAI-1 & 1.45 & $-3.64,6.55$ & & & & & & \\
\hline MTHFR A1298C & -0.36 & $-5.63,4.92$ & & & & & & \\
\hline MTHFR C677T & -1.28 & $-6.69,4.14$ & & & & & & \\
\hline
\end{tabular}

Abbreviations: $\mathrm{Cl}$, confidence interval; $\beta$, regression coefficient.

Note: Thrombophilia markers (common homozygous vs. other) evaluated in placenta/cord blood in singleton, nonanomalous live births $\leq 42$ weeks' gestation.

${ }^{a}$ Any avascular villi (focal, multifocal, diffuse).

${ }^{\text {b}}$ Thrombophilia modeled as presence of 0 versus 1 or more markers in maternal blood.

${ }^{c}$ Model contains thrombophilia marker, placental variables, and covariates (maternal age, maternal race/ethnicity, paternal age, paternal race/ ethnicity).

${ }^{\mathrm{d}}$ Model contains placental variables and covariates (maternal age, maternal race/ethnicity, paternal age, paternal race/ethnicity).

e Model contains thrombophilia marker and covariates (maternal age, maternal race/ethnicity, paternal age, paternal race/ethnicity).

${ }_{\mathrm{f}}$ Model contains individual thrombophilia markers, placental variables, and covariates (maternal age, maternal race/ethnicity, paternal age, paternal race/ethnicity).

${ }^{9}$ Model contains individual thrombophilia markers and covariates (maternal age, maternal race/ethnicity, paternal age, paternal race/ethnicity). 
Table 7 Odds ratios for small for gestational age ( $<10$ th percentile) as a function of presence of maternal thrombophilia markers and placental characteristics related to maternal vascular malperfusion

\begin{tabular}{|c|c|c|c|c|c|c|c|c|c|c|}
\hline \multirow[t]{2}{*}{ All births } & \multicolumn{2}{|c|}{ Thrombophilia } & \multicolumn{2}{|c|}{$\begin{array}{l}\text { Retroplacental } \\
\text { hematoma }\end{array}$} & \multicolumn{2}{|c|}{$\begin{array}{l}\text { Parenchymal } \\
\text { infarction }^{\mathrm{a}}\end{array}$} & \multicolumn{2}{|c|}{$\begin{array}{l}\text { Intraparenchymal } \\
\text { thrombus }\end{array}$} & \multicolumn{2}{|c|}{$\begin{array}{l}\text { Perivillous/ } \\
\text { intervillous } \\
\text { fibrin/fibrinoid } \\
\text { deposition }\end{array}$} \\
\hline & OR & $95 \% \mathrm{Cl}$ & OR & $95 \% \mathrm{Cl}$ & OR & $95 \% \mathrm{Cl}$ & OR & $95 \% \mathrm{Cl}$ & OR & $95 \% \mathrm{Cl}$ \\
\hline Model $1^{\mathrm{b}, \mathrm{c}}$ & 1.18 & $0.65,2.14$ & 0.42 & $0.13,1.34$ & 1.13 & $0.64,1.99$ & 0.27 & $0.11,0.65$ & 2.26 & $0.63,8.08$ \\
\hline Model $2^{\text {d }}$ & - & - & 0.62 & $0.21,1.80$ & 1.16 & $0.69,1.94$ & 0.28 & $0.13,0.60$ & 2.42 & $0.67,8.69$ \\
\hline Model $3^{\mathrm{b}, \mathrm{e}}$ & 1.23 & $0.69,2.18$ & - & - & - & - & - & - & - & - \\
\hline Model $4^{f}$ & & & 0.37 & $0.09,1.55$ & 1.21 & $0.66,2.22$ & 0.35 & $0.14,0.87$ & 2.08 & $0.47,9.11$ \\
\hline FV Leiden & 1.43 & $0.33,6.11$ & & & & & & & & \\
\hline FII Prothrombin & 0.82 & $0.20,3.30$ & & & & & & & & \\
\hline PAl-1 & 1.12 & $0.66,1.88$ & & & & & & & & \\
\hline MTHFR A1298C & 0.96 & $0.56,1.65$ & & & & & & & & \\
\hline MTHFR C677T & 1.18 & $0.69,2.03$ & & & & & & & & \\
\hline Model $5^{9}$ & & & - & - & - & - & - & - & - & - \\
\hline FV Leiden & 1.63 & $0.48,5.52$ & & & & & & & & \\
\hline Fll Prothrombin & 0.73 & $0.19,2.76$ & & & & & & & & \\
\hline PAI-1 & 1.06 & $0.64,1.75$ & & & & & & & & \\
\hline MTHFR A1298C & 1.00 & $0.60,1.66$ & & & & & & & & \\
\hline MTHFR C677T & 1.16 & $0.69,1.94$ & & & & & & & & \\
\hline
\end{tabular}

Abbreviations: $\mathrm{Cl}$, confidence interval; OR, odds ratio.

Note: Thrombophilia markers (common homozygous vs. other) evaluated in maternal blood in singleton, nonanomalous live births $\leq 42$ weeks' gestation.

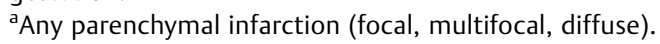

${ }^{\mathrm{b}}$ Thrombophilia modeled as presence of 0 versus 1 or more markers in maternal blood.

${ }^{c}$ Model contains thrombophilia marker, placental variables, and covariates (maternal age, maternal race/ethnicity).

${ }^{\mathrm{d}}$ Model contains placental variables and covariates (maternal age, maternal race/ethnicity).

${ }^{\mathrm{e}}$ Model contains thrombophilia marker and covariates (maternal age, maternal race/ethnicity).

${ }^{f}$ Model contains individual thrombophilia markers, placental variables, and covariates (maternal age, maternal race/ethnicity).

${ }^{9}$ Model contains individual thrombophilia markers and covariates (maternal age, maternal race/ethnicity).

may also be associated with placental changes, including parenchymal infarction, intraparenchymal thrombus, and avascular villi. These placental changes do not appear to account for the observed decrease in fetal growth associated with maternal FV Leiden. Other maternal thrombophilia mutations and all fetal thrombophilia mutations evaluated do not appear to affect birth weight percentile. These results were consistent in a subanalysis restricted to term births (results not shown).

The prevalence of each of the five thrombophilia mutations evaluated is slightly lower than that has been reported in the literature for populations of European descent. ${ }^{3,18,19}$ However, this is expected, as the SCRN study was conducted in a diverse population and these mutations are most common in those of European descent. ${ }^{18}$ Similarly, the mean adjusted birth weight percentile of our study population was lower than expected, likely due to the use of Hadlock's growth curves, which are based on middle-class white women. ${ }^{15}$

The measure of association for FV Leiden in our SGA analysis was consistent in magnitude with a systematic review and meta-analysis of 32 studies on the association between maternal FV Leiden mutation and SGA (pooled OR: 1.40; $95 \%$ CI: $1.18,1.67) .^{5}$ Our lack of statistical significance may be due to small sample size. Of the 22 mothers with the FV Leiden mutation, only five delivered SGA infants. Several studies have reported no association between other thrombophilia mutations (FII prothrombin, MTHFR A1298C, and MTHFR C677T) and fetal growth restriction, which is consistent with our results. ${ }^{6,20}$ The consistency of our results with the literature strengthens the likelihood that our observed association with FV Leiden is a real association and not a result of uncontrolled confounding or another potential bias.

One study evaluating placental abnormalities associated with FV Leiden mutation reported that fetal FV Leiden mutation is associated with avascular villi and maternal FV Leiden mutation is associated with increased numbers of syncytial knots and hypervascular villi. ${ }^{21}$ Our study also found that avascular villi were more than twice as common in the presence of fetal FV Leiden mutation. To our knowledge, no studies have considered placental function and characteristics when evaluating the relationship between inherited thrombophilia and fetal growth restriction, and 
Table 8 Odds ratios for small for gestational age ( $<10$ th percentile) as a function of presence of thrombophilia markers in placenta/cord blood and placental characteristics related to fetal vascular malperfusion

\begin{tabular}{|c|c|c|c|c|c|c|c|c|}
\hline \multirow[t]{2}{*}{ All Births } & \multicolumn{2}{|c|}{ Thrombophilia } & \multicolumn{2}{|c|}{$\begin{array}{l}\text { Fetal vascular } \\
\text { thrombi in the } \\
\text { chorionic plate }\end{array}$} & \multicolumn{2}{|c|}{ Avascular villia } & \multicolumn{2}{|c|}{ Edema } \\
\hline & OR & $95 \% \mathrm{Cl}$ & OR & $95 \% \mathrm{Cl}$ & OR & $95 \% \mathrm{Cl}$ & OR & $95 \% \mathrm{Cl}$ \\
\hline Model $1^{\mathrm{b}, \mathrm{c}}$ & 1.28 & $0.73,2.26$ & 1.90 & $0.98,3.72$ & 1.09 & $0.56,2.14$ & 1.64 & $0.19,14.27$ \\
\hline Model $2^{\mathrm{d}}$ & - & - & 1.43 & $0.75,2.74$ & 1.11 & $0.59,2.09$ & 0.36 & $0.07,1.85$ \\
\hline Model $3^{\text {b,e }}$ & 1.32 & $0.74,2.34$ & - & - & - & - & - & - \\
\hline Model $4^{f}$ & & & 1.77 & $0.88,3.59$ & 1.13 & $0.54,2.36$ & 2.58 & $0.25,26.99$ \\
\hline FV Leiden & 0.60 & $0.13,2.76$ & & & & & & \\
\hline Fll prothrombin & 0.41 & $0.05,3.54$ & & & & & & \\
\hline PAI-1 & 0.95 & $0.58,1.54$ & & & & & & \\
\hline MTHFR A1298C & 1.03 & $0.61,1.73$ & & & & & & \\
\hline MTHFR C677T & 1.37 & $0.79,2.35$ & & & & & & \\
\hline Model $5^{9}$ & & & - & - & - & - & - & - \\
\hline FV Leiden & 0.61 & $0.13,2.84$ & & & & & & \\
\hline FIl prothrombin & 0.39 & $0.05,3.33$ & & & & & & \\
\hline PAI-1 & 0.94 & $0.58,1.52$ & & & & & & \\
\hline MTHFR A1298C & 1.03 & $0.62,1.74$ & & & & & & \\
\hline MTHFR C677T & 1.35 & $0.79,2.33$ & & & & & & \\
\hline
\end{tabular}

Abbreviations: $\mathrm{Cl}$, confidence interval; OR, odds ratio.

Note: Thrombophilia markers (common homozygous vs. other) evaluated in placenta/cord blood in singleton, nonanomalous live births $\leq 42$ weeks' gestation.

${ }^{a}$ Any avascular villi (focal, multifocal, diffuse).

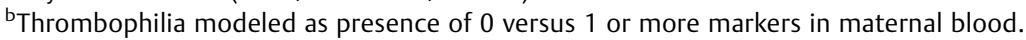

${ }^{c}$ Model contains thrombophilia marker, placental variables, and covariates (maternal age, maternal race/ethnicity, paternal age, paternal race/ ethnicity).

${ }^{d}$ Model contains placental variables and covariates (maternal age, maternal race/ethnicity, paternal age, paternal race/ethnicity).

${ }^{\mathrm{e}}$ Model contains thrombophilia marker and covariates (maternal age, maternal race/ethnicity, paternal age, paternal race/ethnicity).

${ }_{\mathrm{f}}$ Model contains individual thrombophilia markers, placental variables, and covariates (maternal age, maternal race/ethnicity, paternal age, paternal race/ethnicity).

${ }^{9}$ Model contains individual thrombophilia markers and covariates (maternal age, maternal race/ethnicity, paternal age, paternal race/ethnicity).

few studies have considered the role of fetal inherited thrombophilia.

One limitation of this study includes relatively low power due to low prevalence of the placental characteristics of interest and low prevalence of some mutations, particularly FV Leiden and FII prothrombin. This is true even though the SCRN population has a relatively large sample size and had data on placental characteristics and genetic markers on nearly 1,000 live births. In an effort to increase sample size, we evaluated the effect of thrombophilia in all live births with thrombophilia testing (not restricted to those with a placental examination) and our results for maternal FV Leiden were not consistent $(\beta$ : $-2.96 ; 95 \% \mathrm{CI}:-14.50$, $8.58)$. This may also be a result of small sample size. In the total sample, there were 33 births with the maternal FV Leiden mutation and 22 in the sample restricted to those with a completed placental examination. This may be a selection issue, but this seems unlikely given that the proportion of those with maternal FV Leiden was consistent in both samples (2.8\%). Additionally, $93.4 \%$ of women consented to a placental examination and in the majority who did not have a complete examination, the placenta had been previously discarded. Owing to the low prevalence, we were unable to evaluate heterozygotes and homozygotes separately. Additionally, as this was a preliminary study, we did not adjust for multiple testing. We also focused our results on magnitude rather than statistical significance, which is consistent with the American Statistical Association's stance on $p$-values. ${ }^{17}$ These results should be evaluated in populations with a higher prevalence of FV Leiden mutation.

Another limitation is the use of birth weight percentile as an indicator of fetal growth. While fetal growth implies longitudinal measures of growth, birth weight at delivery reflects cumulative growth over the course of the pregnancy. Use of birth weight percentile is advantageous over birth weight because it accounts for variation in size by gestational age. A change in birth weight percentile has a different implication for magnitude of change in actual birth weight 
at different gestational ages. Although not a perfect measure of fetal growth, the use of continuous birth weight percentile is also a strength. Previous studies have dichotomized fetal growth at various cutoffs ( $<5$ th percentile, $<10$ th percentile, $<2$ standard deviations below the mean) or based on abnormal blood flow from Doppler readings. Variations in the definition used may explain the conflicting results. ${ }^{5,22}$ Use of a continuous measure from published norms enhances the comparability and reproducibility of our results.

Additional strengths of this study include standardized protocol for sample collection, storage, and analysis to determine the presence of the thrombophilia markers. Similarly, all study pathologists used a standardized placental examination protocol. Additionally, the SCRN study included a relatively large population that was both geographically and racially diverse.

Maternal FV Leiden appears to negatively affect fetal growth, independent of placental characteristics related to maternal vascular malperfusion. Further research is needed on populations with a higher prevalence of FV Leiden to evaluate this association with improved precision.

\section{Funding}

This work was supported by funding from the Eunice Kennedy Shriver National Institute of Child Health and Human Development, National Institutes of Health, and with supplemental funding from the Office of Research in Women's Health, National Institutes of Health (grants U10-HD045953 (Brown University), U10-HD045925 (Emory University), U10-HD045952 (University of Texas Medical Branch at Galveston), U10-HD045955 (University of Texas Health Sciences Center at San Antonio), UK10HD045944 (University of Utah Health Sciences Center), U10-HD045954 and HHSN275201400001C (RTI International), and 5T32HD052460-10 to A.A.F.). This work was also supported by funding from the Maternal Child Health Bureau, Health Resources and Services Administration (grant T03MC07651 to A.A.F.).

\section{Conflict of Interest}

None declared.

\section{Members of the Study Group}

The Stillbirth Collaborative Research Network-University of Texas Health Science Center at San Antonio: Dr. Donald J. Dudley, Dr. Deborah Conway, Josefine Heim-Hall, Karen Aufdemorte, and Angela Rodriguez; University of Utah School of Medicine: Dr. Robert M. Silver, Dr. Michael W. Varner, and Kristi Nelson; Emory University School of Medicine and Rollins School of Public Health: Dr. Carol J. Rowland Hogue, Dr. Barbara J. Stoll, Janice Daniels Tinsley, Dr. Bahig Shehata, and Dr. Carlos Abromowsky; Brown University: Dr. Donald Coustan, Dr. Halit Pinar, Dr. Marshall Carpenter, and Susan Kubaska; University of Texas Medical Branch at Galveston: Dr. George R. Saade, Dr. Radek Bukowski, Jennifer Lee Rollins, Dr. Hal Hawkins, and Elena Sbrana; RTI International: Dr. Corette B. Parker, Dr.
Matthew A. Koch, Vanessa R. Thorsten, Holly Franklin, and Pinliang Chen; Pregnancy and Perinatology Branch, Eunice Kennedy Shriver National Institute of Child Health and Human Development: Drs. Marian Willinger and Uma M. Reddy; Columbia University Medical Center: Dr. Robert L. Goldenberg.

The Stillbirth Collaborative Research Network Writing Group-Dr. Carol J. R. Hogue (Department of Epidemiology, Rollins School of Public Health, Emory University, Atlanta, Georgia); Dr. Robert L. Goldenberg (Department of Obstetrics and Gynecology, Columbia University Medical Center, New York, New York); Drs. Radek Bukowski and George R. Saade (Department of Obstetrics and Gynecology, University of Texas Medical Branch at Galveston, Galveston, Texas); Dr. Barbara J. Stoll (McGovern Medical School, University of Texas Health Science Center, Houston, Texas); Drs. Marshall Carpenter, Donald Coustan, and Halit Pinar (Division of Maternal-Fetal Medicine, Department of Obstetrics and Gynecology, Brown University School of Medicine, Providence, Rhode Island); Dr. Deborah Conway (Division of Maternal-Fetal Medicine, Department of Obstetrics and Gynecology, University of Texas Health Science Center at San Antonio, San Antonio, Texas); Dr. Donald J. Dudley (Division of Maternal-Fetal Medicine, Department of Obstetrics and Gynecology, University of Virginia, Charlottesville, Virginia); Drs. Robert M. Silver and Michael W. Varner (Division of Maternal-Fetal Medicine, Department of Obstetrics and Gynecology, University of Utah School of Medicine, and Maternal Fetal Medicine Unit, Intermountain Healthcare, Salt Lake City, Utah); Drs. Uma M. Reddy and Marian Willinger (Pregnancy and Perinatology Branch, Eunice Kennedy Shriver National Institute of Child Health and Human Development, Bethesda, Maryland); and Drs. Matthew A. Koch and Corette B. Parker (Statistics and Epidemiology Unit, Health Sciences Division, RTI International, Research Triangle Park, North Carolina).

\section{Acknowledgments}

We acknowledge the contribution of the Stillbirth Collaborative Research Network. We also acknowledge the members of the National Institute of Child Health and Human Development Scientific Advisory and Safety Monitoring Board for their review of the study protocol, materials, and progress, as well as all of the other physicians, study coordinators, and research nurses in the Stillbirth Collaborative Research Network.

\section{References}

1 James AH. Thrombosis in pregnancy and maternal outcomes. Birth Defects Res C Embryo Today 2015;105(03):159-166

2 Davenport WB, Kutteh WH. Inherited thrombophilias and adverse pregnancy outcomes: a review of screening patterns and recommendations. Obstet Gynecol Clin North Am 2014;41(01):133-144

3 American College of Obstetricians and Gynecologists Women's Health Care Physicians. ACOG Practice Bulletin No. 138: inherited 
thrombophilias in pregnancy. Obstet Gynecol 2013;122(03): 706-717

4 Howley HE, Walker M, Rodger MA. A systematic review of the association between factor $\mathrm{V}$ Leiden or prothrombin gene variant and intrauterine growth restriction. Am J Obstet Gynecol 2005; 192(03):694-708

5 Hemsworth EM, O'Reilly AM, Allen VM, Kuhle S, Brock JK; Knowledge Synthesis Group on Determinants of Preterm/LBW Births. Association between factor V Leiden mutation, small for gestational age, and preterm birth: a systematic review and metaanalysis. J Obstet Gynaecol Can 2016;38(10):897-908

6 Franchi F, Cetin I, Todros T, et al. Intrauterine growth restriction and genetic predisposition to thrombophilia. Haematologica 2004;89(04):444-449

7 Pileri P, Franchi F, Cetin I, et al. Maternal and fetal thrombophilia in intrauterine growth restriction in the presence or absence of maternal hypertensive disease. Reprod Sci 2010;17(09):844-848

8 Moll S, Varga EA. Homocysteine and MTHFR mutations. Circulation 2015;132(01):e6-e9

9 Pandey K, Dubay P, Bhagoliwal A, Gupta N, Tyagi G. Hyperhomocysteinemia as a risk factor for IUGR. J Obstet Gynaecol India 2012;62(04):406-408

10 Said JM, Tsui R, Borg AJ, et al. The PAI-1 4G/5G polymorphism is not associated with an increased risk of adverse pregnancy outcome in asymptomatic nulliparous women. J Thromb Haemost 2012;10(05):881-886

11 Glueck CJ, Phillips H, Cameron D, et al. The 4G/4G polymorphism of the hypofibrinolytic plasminogen activator inhibitor type 1 gene: an independent risk factor for serious pregnancy complications. Metabolism 2000;49(07):845-852

12 Silver RM, Saade GR, Thorsten V, et al. Factor V Leiden, prothrombin G20210A, and methylene tetrahydrofolate reductase mutations and stillbirth: the Stillbirth Collaborative Research Network. Am J Obstet Gynecol 2016;215(04):468.e1-468.e17
13 Souza PC, Alves JA, Maia SM, Araujo Júnior E, Santana EF, Silva Costa FD. The $4 \mathrm{G} / 4 \mathrm{G}$ polymorphism of the plasminogen activator inhibitor-1 (PAI-1) gene as an independent risk factor for placental insufficiency, which triggers fetal hemodynamic centralization. Ceska Gynekol 2015;80(01):74-79

14 Parker CB, Hogue CJ, Koch MA, et al; Stillbirth Collaborative Research Network. Stillbirth Collaborative Research Network: design, methods and recruitment experience. Paediatr Perinat Epidemiol 2011;25(05):425-435

15 Hadlock FP, Harrist RB, Martinez-Poyer J. In utero analysis of fetal growth: a sonographic weight standard. Radiology 1991;181(01): 129-133

16 Pinar H, Koch MA, Hawkins H, et al. The Stillbirth Collaborative Research Network (SCRN) placental and umbilical cord examination protocol. Am J Perinatol 2011;28(10):781-792

17 SUDAAN Language Manual, Volumes 1 and 2, Release 11.0. Research Triangle Park, NC: Research Triangle Institute; 2012

18 Franco RF, Reitsma PH. Genetic risk factors of venous thrombosis. Hum Genet 2001;109(04):369-384

19 Peng F, Labelle LA, Rainey BJ, Tsongalis GJ. Single nucleotide polymorphisms in the methylenetetrahydrofolate reductase gene are common in US Caucasian and Hispanic American populations. Int J Mol Med 2001;8(05):509-511

20 Said JM, Higgins JR, Moses EK, Walker SP, Monagle PT, Brennecke SP. Inherited thrombophilias and adverse pregnancy outcomes: a case-control study in an Australian population. Acta Obstet Gynecol Scand 2012;91(02):250-255

21 Rogers BB, Momirova V, Dizon-Townson D, et al. Avascular villi, increased syncytial knots, and hypervascular villi are associated with pregnancies complicated by factor $\mathrm{V}$ Leiden mutation. Pediatr Dev Pathol 2010;13(05):341-347

22 Kist WJ, Janssen NG, Kalk JJ, Hague WM, Dekker GA, de Vries JI. Thrombophilias and adverse pregnancy outcome - A confounded problem!. Thromb Haemost 2008;99(01):77-85 